\title{
Genome-wide identification, classification and expression of lipoxygenase gene family in pepper
}

\author{
Sandeep J. Sarde ${ }^{1} \cdot$ Abhishek Kumar $^{2} \cdot$ Rahima N. Remme $^{1} \cdot$ Marcel Dicke $^{1}[$
}

Received: 18 June 2018 / Accepted: 5 October 2018 / Published online: 13 October 2018

(c) The Author(s) 2018

\begin{abstract}
Key message Lipoxygenases mediate important biological processes. Through comparative genomics, domain-scan analysis, sequence analysis, phylogenetic analysis, homology modelling and transcriptional analysis the lipoxygenase gene family of pepper (Capsicum annuum) has been identified.

Abstract Lipoxygenases (LOXs) are non-heme, iron-containing dioxygenases playing a pivotal role in diverse biological processes in plants, including defence and development. Here, we exploited the recent sequencing of the pepper genome to investigate the $L O X$ gene family in pepper. Two LOX classes are recognized, the 9- and 13-LOXs that oxygenate lipids at the 9th and 13th carbon atom, respectively. Using two main in-silico approaches, we identified a total of eight LOXs in pepper. Phylogenetic analysis classified four LOXs (CaLOX1, CaLOX3, CaLOX4 and CaLOX5) as 9-LOXs and four (CaLOX2, CaLOX6, CaLOX7 and CaLOX8) as 13-LOXs. Furthermore, sequence similarity/identity and subcellular localization analysis strengthen the classification predicted by phylogenetic analysis. Pivotal amino acids together with all domains and motifs are highly conserved in all pepper LOXs. Expression of 13-LOXs appeared to be more dynamic compared to 9-LOXs both in response to exogenous JA application and to thrips feeding. Bioinformatic and expression analyses predict the putative functions of two 13-LOXs, CaLOX6 and CaLOX7, in the biosynthesis of Green Leaf Volatiles, involved in indirect defence. The data are discussed in the context of LOX families in solanaceous plants and plants of other families.
\end{abstract}

Keywords Pepper · Lipoxygenases (LOXs) · Phylogenetic analysis · Gene transcription · Sequence analyses · Defence

\section{Introduction}

Lipoxygenases (EC 1.13.11.12) are non-heme, iron-containing dioxygenases ubiquitously present in plants, animals and fungi (Brash 1999). In plants, lipoxygenases (LOXs) are well-known to be involved in several plant processes like tuber development, seed germination, fruit ripening and most importantly in plant defences (Bailly et al. 2002; Barry and Giovannoni 2007; Feussner and Wasternack 2002;

Electronic supplementary material The online version of this article (https://doi.org/10.1007/s11103-018-0785-y) contains supplementary material, which is available to authorized users.

Marcel Dicke

marcel.dicke@wur.nl

1 Laboratory of Entomology, Wageningen University, P.O. Box 16, 6700 AA Wageningen, The Netherlands

2 Division of Molecular Genetic Epidemiology, German Cancer Research Center, Heidelberg, Germany
Kessler 2004; Kolomiets et al. 2001; Yan et al. 2013). Upon insect or pathogen attack, LOXs oxidize polyunsaturated fatty acids (PUFAs) (linoleic acid, $\alpha$-linolenic acid and arachidonic acid) constituting a (Z,Z)-1,4-pentadiene structural unit and catalyzing it into conjugated hydro-peroxides such as oxylipins (Brash 1999; Feussner and Wasternack 2002; Shibata and Axelrod 1995). Oxylipins such as jasmonates, green leaf volatiles (GLVs) and recently discovered death acids, are known for their roles in defence against herbivorous insects and pathogens (Allmann et al. 2010; Bell et al. 1995; Christensen et al. 2015; Losvik et al. 2017; Shen et al. 2014; Yan et al. 2013). Jasmonates and GLVs are 13-LOXderived products involved in direct and indirect defences, respectively. In indirect defence, GLVs play a pivotal role in the attraction of natural enemies of the herbivores (ul Hassan et al. 2015). Death acids (10-OPDA, 10-oxo-11-phytodienoic acid, and 10-OPEA, 10-oxo-11-phytoenoic acid) are 9-LOX-derived products that in maize (Zea mays) accumulate upon southern leaf blight (Cochliobolus heterostrophus) 
infection resulting in the hampering of growth of fungi and herbivorous insects (Christensen et al. 2016, 2015).

Plant LOXs are primarily classified into two major classes, 9- and 13-LOXs, based on their positional specificity to oxygenate linoleic acids (LAs) (Feussner and Wasternack 2002). Moreover, LOXs are also classified as Type- 1 and Type-2 based on their primary structure and sequence similarity. LOXs having high sequence similarity (>75\%) among themselves and having no plastidic transit peptide are classified as Type-1, whereas LOXs with moderate sequence similarity ( $>35 \%)$ and possessing a plastidic transit peptide are classified as Type-2 (Brash 1999; Feussner and Wasternack 2002). All Type-2 LOXs known at present are 13-LOXs, whereas Type-1 LOXs include both 9- and 13-LOXs (Feussner and Wasternack 2002).

Information on LOXs from several plants has been reported. The Arabidopsis genome comprises a total of six LOXs (AtLOX1-AtLOX6) (Umate 2011). AtLOX1 is upregulated in leaves upon pathogen attack and stress-related hormones (Melan et al. 1993); AtLOX2 is involved in jasmonic acid (JA) biosynthesis (Bell et al. 1995); AtLOX3 and AtLOX4 are essential for flower growth and male fertility (Caldelari et al. 2011); AtLOX5 is important for lateral root development and defence responses (Vellosillo et al. 2007) and AtLOX6 is expressed in roots and involved in JA synthesis (Grebner et al. 2013). Among solanaceous plants, different numbers of LOXs are reported in tomato, potato and tobacco. In tomato, SILOXA (TomLOXA) and SILOXB (Tom$\angle O X B$ ) are induced during fruit ripening (Ferrie et al. 1994; Griffiths et al. 1999); SILOXC (TomLOXC) participates in production of flavour compounds resulting from fatty acids (Chen et al. 2004); SILOXD (TomLOXD) is involved in wound-induced JA biosynthesis, enhancing resistance against herbivores and pathogens (Yan et al. 2013); SILOXE (TomLOXE) is expressed in breaker fruit (Chen et al. 2004) and SILOXF (TomLOXF) enhances systemic resistance stimulated by Pseudomonas putida BTP1(Mariutto et al. 2011). In tobacco, NaLOX1 codes for a 9-LOX and is specifically expressed in roots (Allmann et al. 2010); NaLOX2 is involved in biosynthesis of GLVs (Allmann et al. 2010; VanDoorn et al. 2010); and NaLOX3 is involved in JA biosynthesis (Halitschke and Baldwin 2003; Kessler 2004). Furthermore, in potato, StLOXH1 mediates the biosynthesis of volatile C6-aldehydes (GLVs) involved in defence (Leon et al. 2002) and StLOXH3 is involved in the JA biosynthetic pathway (Royo et al. 1996). Knowledge on LOXs has also been presented in grapevine (Podolyan et al. 2010), kiwifruit (Zhang et al. 2006), rice (Umate 2011), apple (Vogt et al. 2013), soybean (Shin et al. 2008), cucumber (Liu et al. 2011), and olive (Padilla et al. 2009, 2012).

Pepper (Capsicum annuum) is an economically important crop worldwide. It is used e.g. as food, spice and in pharmacology. There are many biotic and abiotic factors constraining pepper production (Kulkarni and Phalke 2009; Kurunc et al. 2011; Pakdeevaraporn et al. 2005; Shipp et al. 1998). Despite increasing commercial significance of pepper, the molecular mechanisms underlying different plant processes are still unknown. For instance, to develop resistance against pathogens and insects, identifying genes involved in different defence mechanisms in pepper is important.

To date, no comprehensive knowledge on the pepper $L O X$ gene family is available. One 9-LOX, CaLOX1, involved in defence and cell-death responses against pathogens has been reported (Hwang and Hwang 2010). Recently, a second member of the LOX gene family (CaLOX2; Capana03g000103) was identified, playing a role in JA-regulated defence against Western flower thrips (Frankliniella occidentalis) (Sarde et al. 2018). Therefore, there is a need of a genome-wide survey of the $L O X$ gene family of pepper. Here, we performed comparative genomics and domain-scan analyses for identification and classification of the $L O X$ gene family in pepper. To investigate the conservation levels of pepper LOXs compared to known LOXs of other plant species, we subjected pepper LOXs to sequence analysis, phylogenetic analysis and homology modelling. Furthermore, to investigate the role of pepper LOXs in defence mechanisms, we examined their expression upon two treatments: exogenous JA application and exposure to feeding by a natural inducer of JA, the cell-content feeding insect Western flower thrips (WFT). WFT was selected because it is a major pest on pepper and well-known to induce JA signaling (Hickman et al. 2017; Steenbergen et al. 2018). The resulting data provide insights into putative functions of these genes in pepper.

\section{Materials and methods}

\section{Sequence acquisition and identification of pepper LOXs}

Protein sequences of tomato (Solanum lycopersicum) lipoxygenases were obtained from the Ensembl Plants database (http://www.ensembl.org) (Yates et al. 2016). LOX sequences from Brassica oleracea, Brassica napus, Brassica rapa, Arabidopsis thaliana, Nicotiana attenuata, Nicotiana tabacum, Solanum tuberosum, Zea mays and Actindia deliciosa, were downloaded from NCBI (http://www.ncbi.nlm. nih.gov/). Oryza sativa and Cucumis melo LOX sequences were retrieved from the Rice Genome Annotation Project (http://rice.plantbiology.msu.edu/) and the Melonomics database (http://melonomics.net/), respectively. Two main approaches were used for the identification of the pepper LOX gene family. First, BLAST searches were performed locally on the Capsicum annuum L. Zunla-1 proteome (Qin et al. 2014) using Tomato LOX proteins as queries. Second, 
the Capsicum annuum L. Zunla-1 proteome was entirely analyzed for the presence of lipoxygenase gene family signature domains, LOX and PLAT/LH2 (polycystin-1, lipoxygenase, $\alpha$-toxin domain or lipoxygenase homology) using the Pfam database (v27.0) in the CLC Bioworkbench (https ://www.qiagenbioinformatics.com/).

\section{Sequence alignment of lipoxygenases}

Alignment of LOX protein sequences was performed using the MUSCLE tool (Edgar 2004) with default settings. Editing and visualization of alignment was produced in GENEDOC (Nicholas et al. 1997). Sequence logos of conserved regions in pepper LOX proteins were generated by Weblogo 3.3 (Crooks et al. 2004).

\section{Phylogenetic analysis of plant LOXs}

Seventy-two plant LOX protein sequences were analyzed, including one known pepper LOX, CaLOX1(L) $(L$ stands for 'literature') (Hwang and Hwang 2010) and eight pepper LOXs identified in the present study. A Maximum likelihood tree using WAG-model (Hall 2013), with 1000 bootstrap replicates was generated using MEGA 7.0 (Kumar et al. 2016). The tree was edited with the Figtree tool (http://tree. bio.ed.ac.uk/software/figtree/).

\section{Sequence analysis and identification of conserved sequences}

Conserved sequences and pivotal amino acids were identified by manual observations on pepper LOX alignments in GENEDOC (Nicholas et al. 1997). Molecular weight and isoelectric point of pepper LOX proteins were calculated by protein isoelectric point calculator (Kozlowski 2016). Subcellular localization analysis was performed using TargetP 1.1 (http://www.cbs.dtu.dk/services/TargetP/).

\section{Homology modeling of CaLOX1 and CaLOX2 protein}

We generated a protein structural model of CaLOX1 and CaLOX2 using the I-TASSER (Roy et al. 2010) database and the resulting model was visualized with YASARA (Krieger et al. 2002).

\section{Plant growth conditions, thrips rearing and bioassays}

Sweet pepper [Capsicum annuum (Mandy variety, Rijk Zwaan (De Lier, The Netherlands))] plants were grown in a greenhouse at $23-25{ }^{\circ} \mathrm{C}, 70 \pm 10 \%$ relative humidity and $16 \mathrm{~L}: 8 \mathrm{D}$ photoperiod. Four-week-old plants were used in the experiments for both treatments. Western flower thrips
(WFT; Frankliniella occidentalis) were reared on bean pods (Phaseolus vulgaris) in a climate-controlled cabinet $\left(25 \pm 2{ }^{\circ} \mathrm{C}, 70 \pm 10 \%\right.$ relative humidity, L16:8D photoperiod). For thrips treatment in the gene expression experiment, five 2nd instar thrips larvae were placed in clip cages and used for infestation on one of the first two true leaves. Empty clip cages were used on control plants for each time point. Samples were harvested at $0,2,4,6,8,10$ and $24 \mathrm{~h}$ post infestation, frozen in liquid nitrogen and stored at $-80^{\circ} \mathrm{C}$.

\section{RNA extraction and qRT-PCR}

Transcriptional responses of pepper LOXs in response to JA treatment $(100 \mu \mathrm{M})$ and thrips feeding were assessed by qRT-PCR. For JA-treatment, plants were dipped in $100 \mu \mathrm{M}$ of JA (treatment) or mock-treated with water (control), both mixed with $0.1 \%$ of Tween 20 . One of the first two true leaf samples were harvested at $0,0.5,1,2,3,6,8,10$ and $24 \mathrm{~h}$ post JA application, frozen in liquid nitrogen and stored at $-80{ }^{\circ} \mathrm{C}$. For both treatments (JA and thrips), control samples were harvested at each time point to rule out the effect of circadian rhythm on the expression of LOX genes. Four to five biological replicates (individual plants) were harvested and analysed for each time point and treatment. Each biological replicate comprises one individual plant. Bioline kit (ISOLATE II RNA Plant Kit), in accordance to its protocol, was used for RNA extraction. cDNA synthesis was executed with $1 \mu \mathrm{g}$ of total RNA with Bio-Rad iScript cDNA synthesis kit. For qPCR, a reaction mixture comprising of $12.5 \mu \mathrm{l}$ of SYBR Green (Bioline), $1 \mu \mathrm{l}(10 \mu \mathrm{M})$ of forward and reverse primers, $5.5 \mu \mathrm{l}$ RNase free-water and $5 \mu \mathrm{l}$ cDNA was used. The data normalization was performed with a reference gene, CaActin. The PCR cycle conditions used were $95{ }^{\circ} \mathrm{C}$ for $3 \mathrm{~min}$, followed by 40 cycles of $95^{\circ} \mathrm{C}$ for $15 \mathrm{~s}$, and $60^{\circ} \mathrm{C}$ for $45 \mathrm{~s}$. Melt curves for each gene were recorded at the end of each cycle. All primers used for qPCR are presented in Supplementary file S1.

Relative gene expression was studied using the geometric mean of Ct (threshold cycles) values (Vandesompele et al. 2002) from the reference gene CaActin using the 2- ${ }^{\Delta \Delta \mathrm{Ct}}$ method (Livak and Schmittgen 2001).

\section{Statistical analysis}

The gene expression data were subjected to a Student's $t$-test.

\section{Results and discussion}

\section{Identification of lipoxygenase gene family in pepper}

A genome-wide search for lipoxygenase genes in pepper was performed by implementing two main approaches: 
homology search and scanning of the pepper proteome for the presence of "lipoxygenase" and "PLAT/LH2" domains. Both approaches resulted in the identification of eight lipoxygenases in the Capsicum annum L. Zunla-1 proteome (Table 1). Several proteins depicting the presence of either one lipoxygenase domain or the PLAT/LH2 domain were excluded from analysis based on arguments of Chen et al. (2015).

The total number of LOXs in pepper (8) is similar to that in tomato (7). This number is also close to the number in Arabidopsis (6) (Umate 2011) and kiwifruit (6) (Zhang et al. 2006), double the number in olive (4) (Padilla et al. 2009, 2012) and much lower than in melon (18) (Zhang et al. 2014), cucumber (23) (Liu et al. 2011) and grapevine (18) (Podolyan et al. 2010). This diverse number of LOXs in different plant species indicates that this gene family has not been conserved during evolution, despite similarities in biochemical functions of the gene family in different plant species (Feussner and Wasternack 2002).

Genomic and proteomic features of the pepper LOX gene family do not differ much (Table 1). At the genomic level, the number of introns varies between 7 and 9, whereas, ORF (Open Reading Frame) length ranges from a minimum of $2379 \mathrm{bp}$ to a maximum of $2748 \mathrm{bp}$. Most of the pepper LOXs are located on Chromosomes 1 and 3, with the exception of CaLOX8 (Capana11g000928) on Chromosome 11. At the protein level, LOX length varied between 792 and 915 aa, the predicted isoelectric point (PI) ranged between 5.4 and 7.5 and the predicted molecular weight of the proteins ranged from 89,959 to $104,131 \mathrm{Da}$. Sequence comparison among pepper LOXs at the protein level shows high sequence identity (33-70\%) and similarity (48-77\%) (Table 2). Taken together, these genomic and proteomic features show a close relation among the pepper $L O X s$, indicative of a gene family.

\section{Phylogenetic analysis of lipoxygenases}

To determine the evolutionary relationship and predict the classification of pepper LOXs, a maximum-likelihood phylogenetic tree with 1000 bootstraps was generated. For this, we used sixty-four previously known plus eight pepper LOX protein sequences from twelve different plant species, comprising monocots and dicots. The tree explicitly categorizes plant LOXs into 9-LOXs, 13-LOXs and uncharacterized LOXs. From the identified eight pepper LOXs, four LOXs (CaLOX1, CaLOX3, CaLOX4 and CaLOX5) are characterized, including the previously described CaLOX1(L) (Hwang and Hwang 2010) into the 9-LOX group and four other LOXs (CaLOX2, CaLOX6, CaLOX7 and CaLOX8) into the 13-LOX group (Fig. 1). Moreover, upon closer examination of the 9- and 13-LOXs major clades, explicit sub-clades of monocot and dicot LOXs are formed indicating

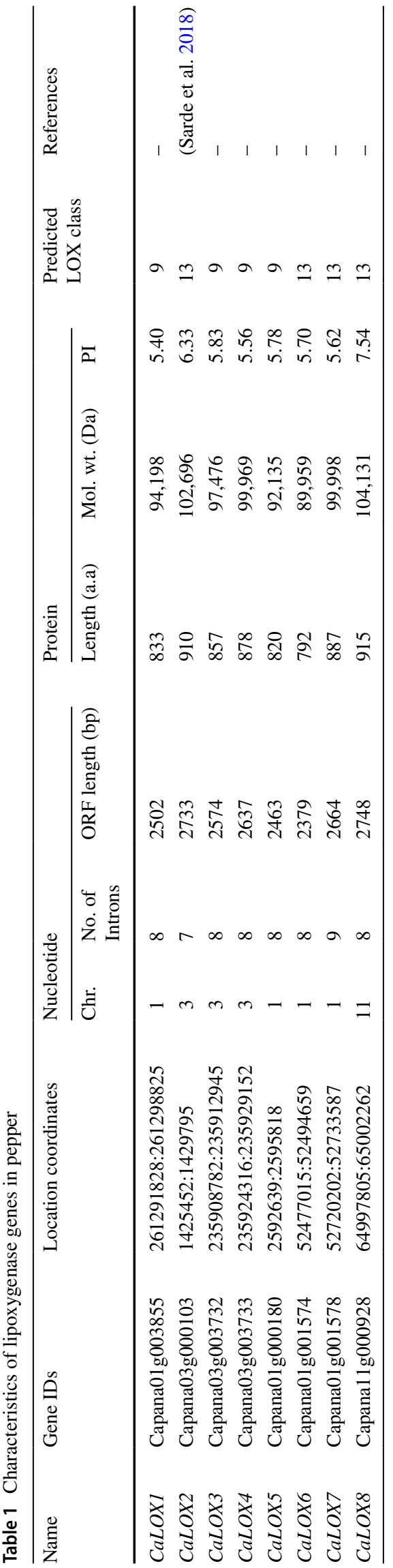


Table 2 Pepper LOX protein identities and similarities (\%)

\begin{tabular}{|c|c|c|c|c|c|c|c|c|c|}
\hline $\begin{array}{l}\text { Protein } \\
\text { IDs }\end{array}$ & CaLOX5 & CaLOX1 & CaLOX3 & CaLOX4 & CaLOX7 & CaLOX6 & CaLOX8 & CaLOX2 & \\
\hline CaLOX5 & & 57 & 52 & 53 & 36 & 33 & 38 & 38 & \multirow{9}{*}{ 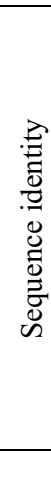 } \\
\hline CaLOX1 & 72 & & 59 & 64 & 39 & 35 & 38 & 40 & \\
\hline CaLOX3 & 70 & 73 & & 70 & 39 & 36 & 37 & 38 & \\
\hline CaLOX4 & 69 & 77 & 83 & & 38 & 35 & 38 & 39 & \\
\hline CaLOX7 & 51 & 56 & 55 & 56 & & 70 & 45 & 45 & \\
\hline CaLOX6 & 48 & 50 & 50 & 48 & 77 & & 42 & 40 & \\
\hline CaLOX8 & 53 & 54 & 54 & 55 & 65 & 59 & & 52 & \\
\hline CaLOX2 & 54 & 57 & 57 & 56 & 62 & 56 & 69 & & \\
\hline & & & & equen & nilarit & & & & \\
\hline
\end{tabular}

High, intermediate and low similarity/identity of genes is shown in green, yellow and red color, respectively
Fig. 1 Phylogenetic analysis of plant lipoxygenases. The evolutionary relationship between pepper and other LOX proteins. The tree was generated by MEGA 7 using Maximum Likelihood method with 1000 bootstraps and viewed in Figtree. The scale bar represents the branch length. Different classes of LOXs are depicted in different colors, 13-LOXs in purple; 9-LOXs in blue; unclassified without color. Identified pepper LOXs are highlighted in red color. Species abbreviations used for phylogeny are as follows. At: Arabidopsis thaliana, Bo: Brassica oleracae, Bn: Brassica napus, Br: Brassica rapa, Sl: Solanum lycopersicum, St: Solanum tuberosum, $\mathrm{Na}$ : Nicotiana attenuata, Nt: Nicotiana tabacum, Ca: Capsicum annuum, Os: Oryza sativa, $\mathrm{Zm}$ : Zea Mays, Cm: Cucumis melo, Ad: Actindia deliciosa

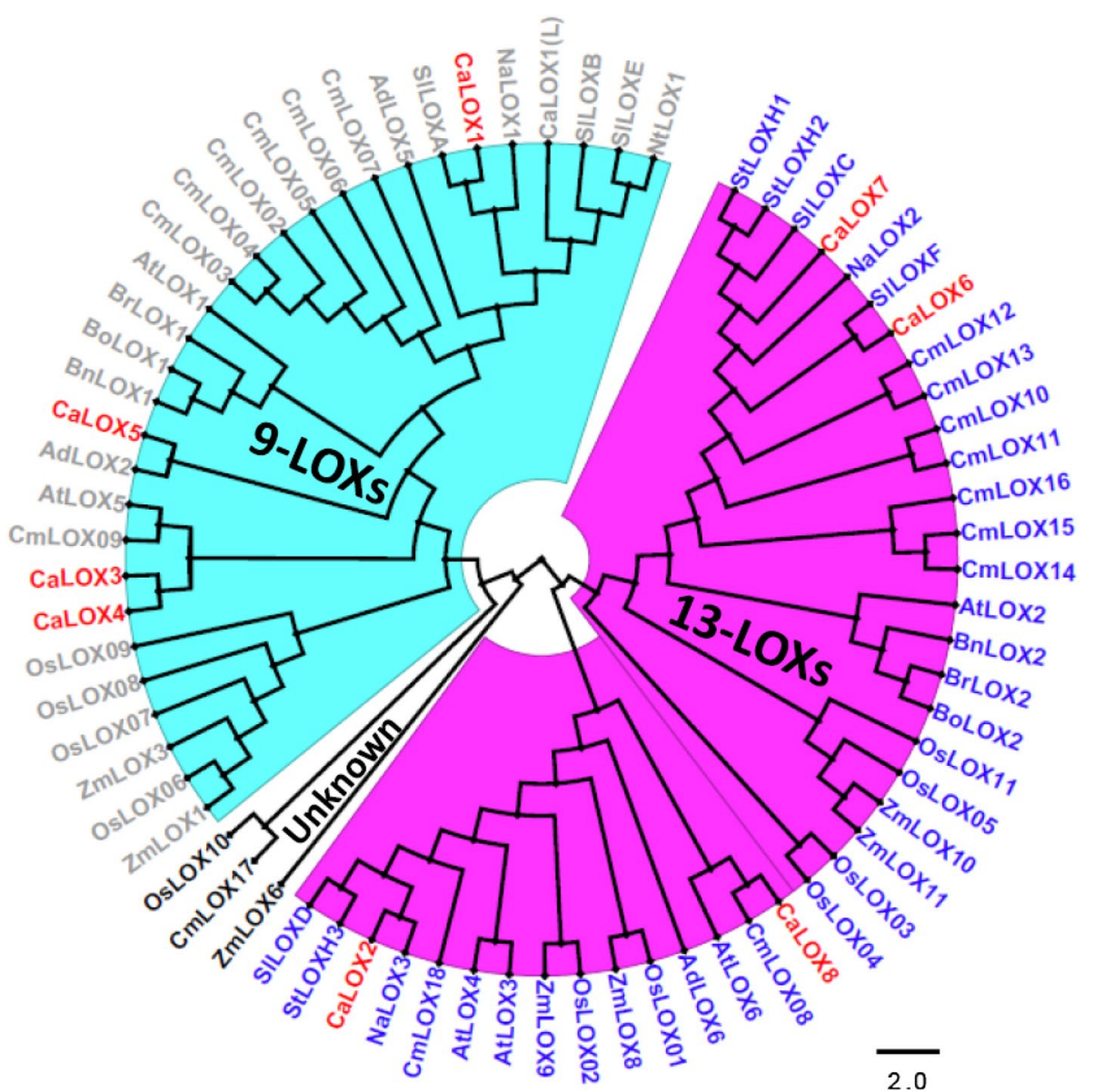

that this gene family has evolved differently in monocots and dicots (Fig. 1).

In the 13-LOX clade, pepper LOXs group with well-characterized Solanaceae 13-LOXs like SILOXD, StLOXH3, NaLOX3, SILOXF, NaLOX2, StLOXH1 and SILOXC (Fig. 1). These clusters or sub-clusters among known LOXs and newly identified LOXs may be useful to predict biochemical features and molecular functions of the newly identified pepper LOXs. CaLOX2 clusters with SILOXD, StLOXH3 and NaLOX3, well-known to be involved in JA biosynthesis (Halitschke and Baldwin 2003; Kessler 2004; Royo et al. 1996; Yan et al. 2013) suggesting that CaLOX2 
has a similar function. This matches with our recent study experimentally confirming that $\mathrm{CaLOX} 2$ is involved in JA biosynthesis upon thrips feeding (Sarde et al. 2018). Virusinduced gene silencing of $C a L O X 2$ led to disruption of the jasmonate pathway resulting in enhanced performance of thrips. CaLOX6 clusters with SILOXF, known to be involved in systemic resistance to Pseudomonas putida BTP1 (Mariutto et al. 2011). CaLOX7 groups with NaLOX2, StLOXH1 and SILOXC. These three LOXs are involved in the biosynthesis of green leaf volatiles (Allmann et al. 2010; Chen et al. 2004; Leon et al. 2002; VanDoorn et al. 2010). CaLOX8 seems to be related to AtLOX6, known to provide resistance against biotic and abiotic stresses through oxylipin biosynthesis in roots (Grebner et al. 2013).

Also in the 9-LOX clade, pepper LOXs cluster with functionally characterized LOXs of other plant species such as AtLOX5, AdLOX2, SILOXA, SILOXB (Fig. 1). AdLOX2, that mediates the generation of $\mathrm{C} 6$ aldehydes in kiwifruit (Zhang et al. 2009), clusters with CaLOX5, suggesting that CaLOX5 has a similar function. CaLOX3 and CaLOX4 form a major clade with AtLOX5 and CmLOX09. AtLOX5 is involved in lateral root development and defence responses (Vellosillo et al. 2007). Additionally, relatedness of CaLOX3 and CaLOX4 to each other, suggests that they may be isoforms mediating the same biological process. Furthermore, clustering together of identified CaLOX1 from Capsicum annuum Zunla-1 proteome (Qin et al. 2014) and known CaLOX1(L) reflects their similarity/relatedness, suggesting them to be the same protein. Hwang and Hwang (2010) identified $\operatorname{CaLOXI}(L)$ independently from cDNA clones and reported it to be involved in defence and cell-death responses against pathogens. Furthermore, CaLOX1 identified here and the previously reported CaLOX1(L) (Hwang and Hwang 2010) cluster with LOXs like SILOXA and SILOXB, two LOXs that are up-regulated in ripening tomato fruits (Ferrie et al. 1994; Griffiths et al. 1999). Nevertheless, taken together, the predicted functions of pepper LOXs require further experimental validation to characterize their molecular functions, as reported for CaLOX1(L) and CaLOX2 (Hwang and Hwang 2010; Sarde et al. 2018).

Finally, the reported uncharacterized LOXs like OsLOX10, CmLOX17 and ZmLOX6 clearly form an outgroup from the 9- and 13-LOXs (Cao et al. 2016; Liu et al. 2011; Zhang et al. 2014).

\section{Sequence analysis consolidates phylogenetic classification of pepper LOXs}

The lipoxygenase family of pepper (CaLOX1-CaLOX8) is highly conserved with variable sequence identities and similarities with each other (Table 2). It is known that, based on degree of sequence similarity and presence/absence of chloroplast-transit peptide, LOXs are classified into Type-1 or Type-2 (Feussner and Wasternack 2002; Porta and RochaSosa 2002). Type-1 LOXs show high sequence similarity $(>75 \%)$ in the absence of a chloroplast-transit peptide; in contrast, Type-2 LOXs show low sequence similarity and the presence of a chloroplast-transit peptide. The pepper LOXs CaLOX1 and CaLOX3, CaLOX4 and CaLOX5, exhibit high sequence similarity $(>70 \%)$ and identity $(>52 \%)$ with each other compared to other LOXs. In contrast, CaLOX2 and CaLOX6, CaLOX7 and CaLOX8 show low sequence similarity among themselves with the exception of CaLOX6 and CaLOX7. CaLOX6 and CaLOX7 show high sequence similarity and identity among themselves, but not when compared to the rest of the LOXs, suggesting that CaLOX6 and $\mathrm{CaLOX} 7$ are isoforms of each other. Furthermore, the presence of a chloroplast-transit peptide in sequences of CaLOX2 and CaLOX6, CaLOX7 and CaLOX8 suggests that they are localized in the chloroplast. Collectively, sequence similarity and sub-cellular localization analysis indicates classification of CaLOX1, CaLOX3, CaLOX4 and CaLOX5 into Type- 1 and CaLOX2, CaLOX6, CaLOX7 and CaLOX8 into Type-2.

Furthermore, plant LOXs are also classified into 9- and 13-LOXs, based on their positional specificity of action on the substrate (Feussner and Wasternack 2002). The presence of Phe608/His608 or Val608 residue predicts LOX activity as 13- or 9-LOX, respectively. Multiple sequence alignment of all pepper LOXs clearly shows the occurrence of valine in CaLOX 1 and CaLOX3, CaLOX 4 and CaLOX5 classifying them as 9-LOXs and phenylalanine in CaLOX2 and CaLOX6, CaLOX7 and CaLOX8 classifying them as 13-LOXs (Fig. S1). This agrees with the observation that all Type-2 LOXs identified so far are 13-LOXs (Feussner and Wasternack 2002).

Therefore, both classification methods provide consensus on distribution of pepper LOXs into different classes, thus consolidating our methodology and predictions. Moreover, it also suggests to use the parameters from both approaches in the future for classification of plant lipoxygenases.

\section{High conservation of motifs and pivotal amino acids}

Lipoxygenases are characterized by the presence of a 38-residue representative sequence, a substrate-binding domain, an oxygen binding domain and a C-terminal motif (Padilla et al. 2009, 2012). The highly representative 38-residue sequence in lipoxygenases is important for stability of lipoxygenases. In addition, the enzymatic activity or efficiency of lipoxygenases can be severely affected if any of the residues of this sequence is substituted (Chen et al. 2015). This sequence is highly conserved in all the predicted pepper LOXs (Fig. 2a, e, f). Also, the other motifs like substrate binding, oxygen binding and the $\mathrm{C}$-terminal are conserved among all pepper LOXs (Fig. 2a-f). 
(A) Motif-I

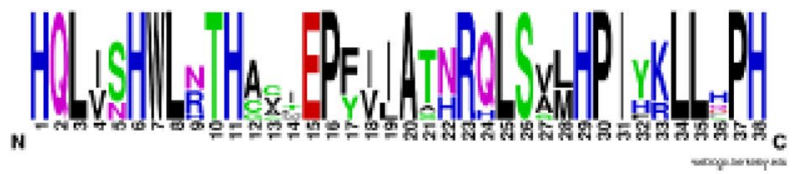

Representative 38-residue

(B) Motif-II

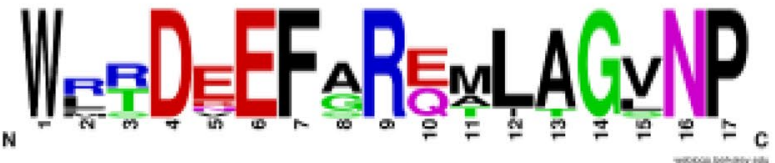

Substrate-binding

(C) Motif-III

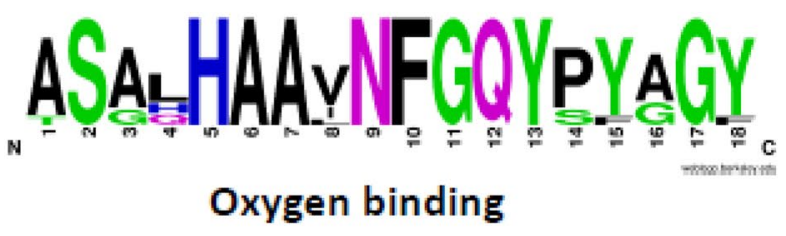

(D) Motif-IV

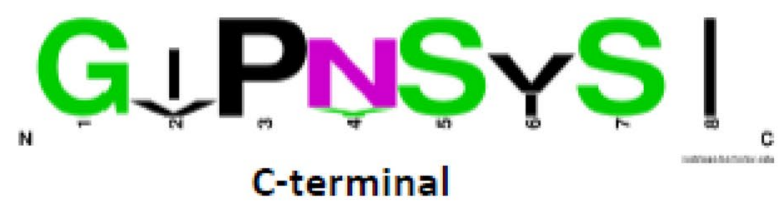

(E) CaLOX1

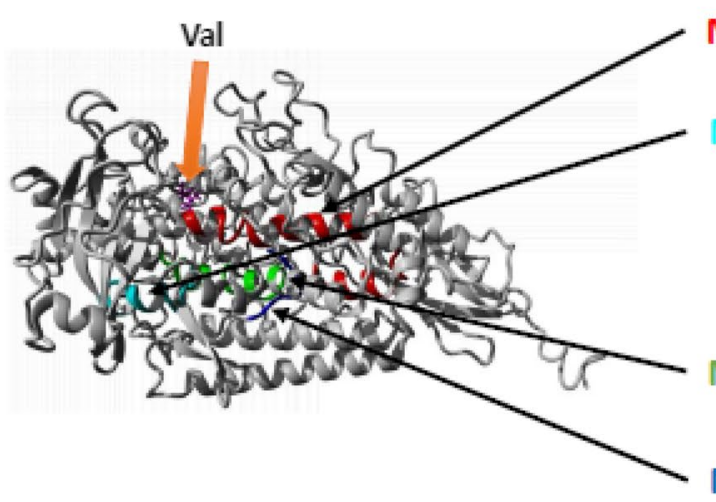

Fig. 2 Conservation of sequence motifs in pepper lipoxygenases (ad) and protein models of CaLOX1 and CaLOX2 (e-f). Highly representative 38-residue motif (a), substrate binding (b), oxygen binding (c) and C-terminal (d) motifs are highly conserved. Protein model of a 9-LOX CaLOX1 (e) and 13-LOX CaLOX2 (f) depicting conser-

Among the conserved amino acids, the three histidine residues (including two from the representative 38-residue sequence) His499, His504, His690 with Asn694 and Ile839 are identified to be vital for binding to non-heme iron (Boyington et al. 1997; Feussner and Wasternack 2002;
(F) CaLOX2

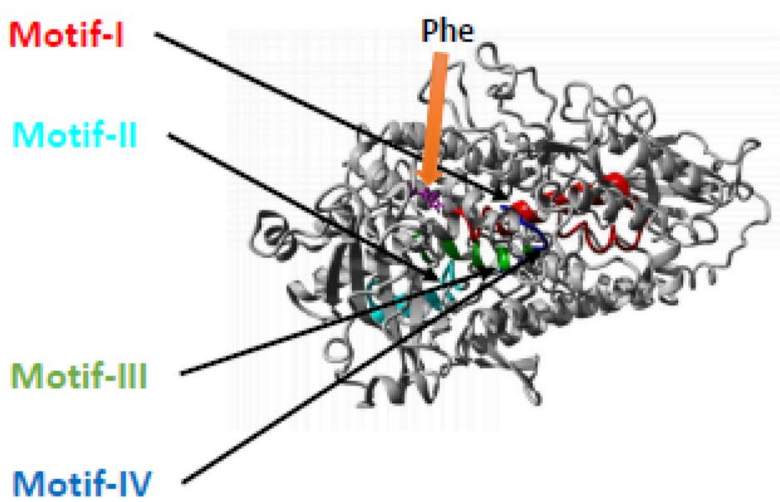

vation of highly representative 38-residue (red), substrate binding (cyan), oxygen binding (green) and C-terminal motif (blue) motifs. 13- or 9-LOX activity determinant Phe or Val residue, respectively are depicted

Padilla et al. 2012; Porta and Rocha-Sosa 2002; Steczko et al. 1992). All these five amino acids appear to be conserved in the pepper LOXs (Fig. S1) with an exception for Ile839 in CaLOX7. Substitution of C-terminal isoleucine with any other amino acid except valine led to inactivation 

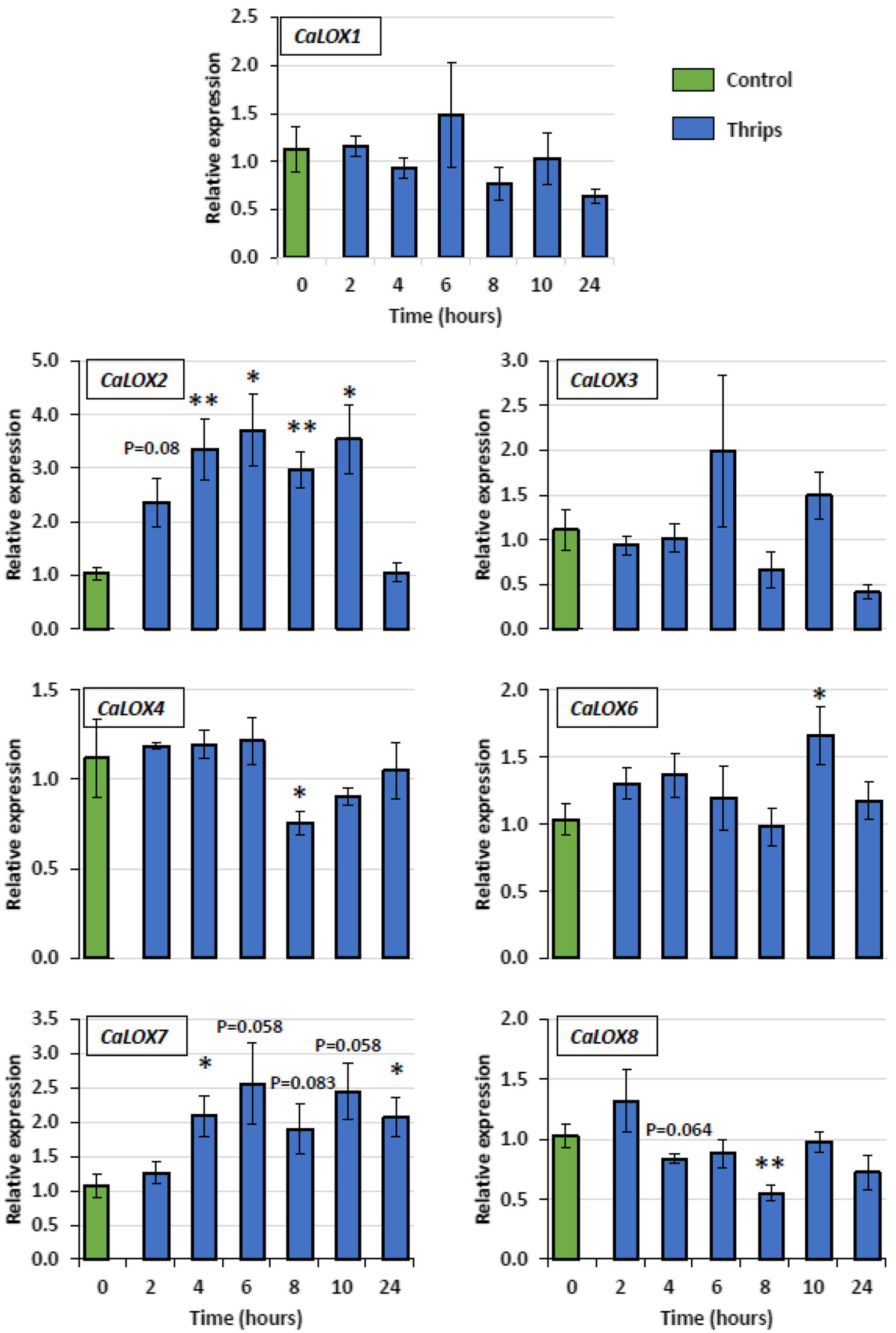
४Fig. 3 Quantitative RT-PCR (RT-qPCR) of pepper lipoxygenase genes in sweet pepper leaves in response to thrips ( $F$. occidentalis) feeding. Five 2 nd instar thrips larvae in a clip cage fed on the first true leaf of 4-week-old pepper plants. Clip cages without thrips were used on control plants. Expression of the housekeeping gene CaActin was used to normalize the expression level of each $L O X$ gene at each time point. Relative expression compared to the control for the same time point is presented. Bars represent means \pm SE of $4-5$ biological replicates. Bars marked with asterisks indicate significant differences (Student's $t$-test) to corresponding control samples for the same time point, $* \mathrm{P}<0.05, * * \mathrm{P}<0.01$. For bars without asterisk or $\mathrm{P}$ value, the $P$ value is $>0.10$

of lipoxygenases, whereas, substitution with valine had positive consequences for enzymatic activity (Chen et al. 1994). Therefore, the absence of a C-terminal motif and the presence of Ile839, essential for non-heme iron binding, leads us to suggest that CaLOX7 may have an altered enzymatic activity. Moreover, the conserved Val608 or Phe608/His608 residue that are indicative for 9- or 13-LOX activity, respectively (Hornung et al. 1999; Padilla et al. 2009, 2012; Sloane et al. 1991), are found highly conserved in pepper LOXs (Fig. 2e, f and Fig. S1). The determinant residues for inverse substrate orientation and S-stereospecificity of LOXs, Arg and Ala, respectively (Coffa and Brash 2004; Hornung et al. 1999) are well-conserved as well in pepper LOXs (Supplemental Fig. S1). Taken together, the conservation of motifs and pivotal amino acids suggests that functions of pepper LOXs are conserved to their respective homologs in other plant species.

\section{Expression pattern of lipoxygenases upon JA application and thrips feeding}

qRT-PCR was performed to investigate the expression dynamics of pepper LOXs over time upon thrips feeding or exogenous JA application. Upon thrips feeding, two 13-LOXs (CaLOX2 and CaLOX7) are up-regulated for most of the analyzed time points (Fig. 3). Induction of CaLOX2 occurred after $2 \mathrm{~h}$ of thrips feeding and remained up-regulated. This gene's involvement in JA biosynthesis has been experimentally supported (Sarde et al. 2018). CaLOX7 is significantly up-regulated at $4 \mathrm{~h}$ after the start of feeding and remained up-regulated throughout the period suggesting that it may have a role in defence. CaLOX6 is significantly up-regulated after $10 \mathrm{~h}$ of feeding. In contrast, all other LOXs, i.e. CaLOX1, CaLOX3, CaLOX4 and CaLOX8 did not show induction over time (Fig. 3). CaLOX4 and CaLOX8 are significantly down-regulated after $8 \mathrm{~h}$ of thrips feeding. CaLOX5 expression is not shown due to its unstable expression resulting in a high degree of variation. This instability of CaLOX5 expression was also confirmed by its expression pattern in an RNA-seq dataset (Sarde et al. unpublished data).
In Arabidopsis, it is well-known that $L O X$ expression is triggered following application of JA due to presence of a positive feedback loop that amplifies JA responses (Hickman et al. 2017). Upon exogenous JA application, CaLOX2, known to be involved in JA biosynthesis (Sarde et al. 2018), shows significant induction after $2 \mathrm{~h}$ which was maintained until $6 \mathrm{~h}$ after JA application with exception at $3 \mathrm{~h}$ (Fig. 4). This instant up- and downregulation of CaLOX2, suggests involvement of some feedback mechanism in JA-biosynthetic pathway. CaLOX6 and CaLOX7 are also upregulated upon JA application. Both of them exhibit high expression levels at similar timepoints i.e., $8 \mathrm{~h}$ and $24 \mathrm{~h}$ after JA application. In contrast, the other $L O X$ genes, i.e. CaLOXI, $C a L O X 3, C a L O X 4$ and $C a L O X 8$, were not up-regulated at any time point, but exhibited down-regulation at several time points (Fig. 4).

In general, the 9-LOXs in pepper (CaLOX1, CaLOX3 and CaLOX4) did not show any induction, but rather down-regulation at certain time points in both treatments, i.e. JA application and thrips feeding. This fits to the fact that 9-LOXs are especially involved in functions such as plant-pathogen interactions, storage of proteins and tuber development (Feussner and Wasternack 2002). In contrast, the 13-LOXs were more responsive to both treatments, except CaLOX8. Similarity of CaLOX7 to NaLOX2 and SILOXC (Fig. 1), both known to be involved in the biosynthesis of green leaf volatiles (GLVs) (Allmann et al. 2010; Chen et al. 2004; Shen et al. 2014; VanDoorn et al. 2010), and it's up-regulation upon both thrips feeding and JA application (Figs. 3, 4) suggest a role of $\mathrm{CaLOX} 7$ in the biosynthesis of GLVs in pepper. Additionally, in tomato SILOXC-antisense lines, low expression of both SILOXC and SILOXF resulted in decreased levels of $\mathrm{C} 5$ and $\mathrm{C} 6$ leaf volatiles, suggesting a possible synergistic involvement of SILOXC and SILOXF in the biosynthesis of these plant volatiles (Shen et al. 2014). Therefore, the similarity of CaLOX6 to SILOXF (Fig. 1) and its induction upon both JA application and thrips feeding makes it a potential candidate to test for its synergistic role with CaLOX7 in volatile biosynthesis (Figs. 3, 4).

In conclusion, this study has identified and classified eight LOXs in pepper. Phylogenetic analysis classified four LOXs as 9-LOXs (CaLOX1, CaLOX3, CaLOX4 and CaLOX5) and four others as 13-LOXs (CaLOX2, CaLOX6, CaLOX7 and CaLOX8) with predictions of their putative functions. Pepper LOX proteins are highly conserved in all lipoxygenase characteristics. Characterization of CaLOX2 encoding for a LOX that is involved in JA biosynthesis is confirmed by a recent experimental study through a combination of in-silico, transcriptional, behavioural, and chemical analyses plus silencing of CaLOX2 through virus-induced gene silencing (Sarde et al. 2018). For the other LOXs their function remains to be elucidated. High expression levels of 13-LOXs in 

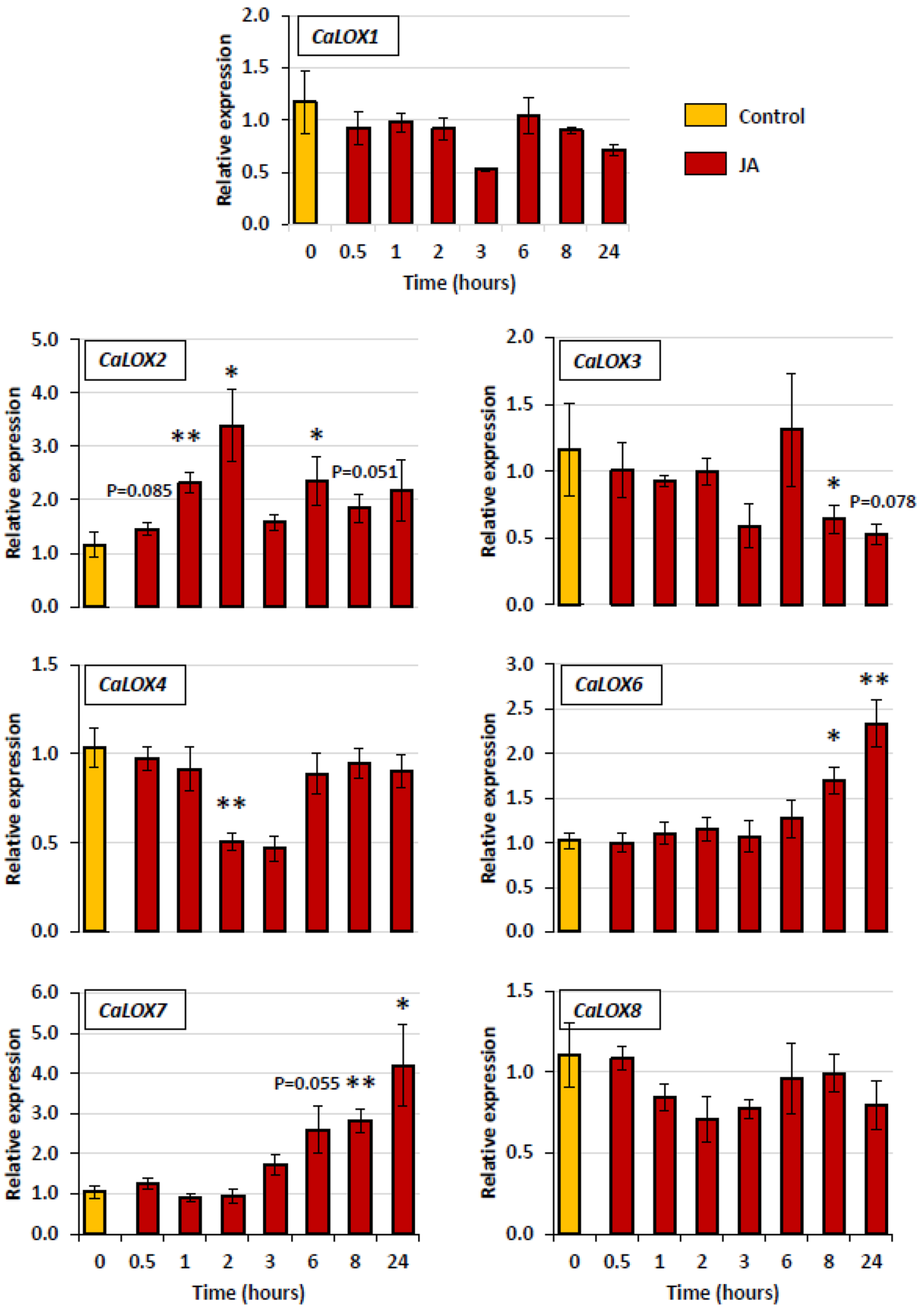

pepper with support of in-silico analysis predict potential candidate genes (CaLOX6 and CaLOX7) that code for enzymes involved in GLV biosynthesis in pepper. Finally,

this comprehensive study provides a pepper $L O X$ genes repository to further elucidate their functional roles in respective biological processes. 
४Fig.4 Quantitative RT-PCR (RT-qPCR) of pepper lipoxygenase genes in sweet pepper leaves in response to exogenous JA application. Pepper plants were dipped in water + Tween 20 (control) or $100 \mu \mathrm{M}$ $\mathrm{JA}+$ Tween20 (treatment). Expression of the housekeeping gene CaActin was used to normalize the expression level of each $L O X$ gene at each time point. Relative expression compared to the control for the same time point is presented. Bars represent means \pm SE of 4-5 biological replicates. Bars marked with asterisks indicate significant differences (Student's $t$-test) to corresponding control samples for the same time point, $* \mathrm{P}<0.05, * * \mathrm{P}<0.01$. For bars without asterisk or $\mathrm{P}$ value, the $P$ value is $>0.10$

Acknowledgements We thank Gerrie Wiegers for helping with the culture of thrips and Wageningen University \& Research experimental farm (Unifarm) for rearing the plants. This work is part of the Perspective programme Green Defences Against Pests that is (partly) financed by the Netherlands Organisation for Scientific Research (NWO, Grant No. 13555) and partly by the breeding companies Keygene, Syngenta and EastWest Seeds through NWO.

Author contributions Conceived and designed the experiments: SJS, AK and MD. Performed the experiments: SJS, RR. Analyzed the data: SJS, RR, AK and MD. Contributed to the writing of the manuscript: SJS, AK and MD.

\section{Compliance with ethical standards}

Competing interests The authors declare that they have no competing interests.

Open Access This article is distributed under the terms of the Creative Commons Attribution 4.0 International License (http://creativeco mmons.org/licenses/by/4.0/), which permits unrestricted use, distribution, and reproduction in any medium, provided you give appropriate credit to the original author(s) and the source, provide a link to the Creative Commons license, and indicate if changes were made.

\section{References}

Allmann S, Halitschke R, Schuurink RC, Baldwin IT (2010) Oxylipin channelling in Nicotiana attenuata: lipoxygenase 2 supplies substrates for green leaf volatile production. Plant Cell Environ 33:2028-2040

Bailly C, Bogatek-Leszczynska R, Come D, Corbineau F (2002) Changes in activities of antioxidant enzymes and lipoxygenase during growth of sunflower seedlings from seeds of different vigour. Seed Sci Res 12:47-55

Barry CS, Giovannoni JJ (2007) Ethylene and fruit ripening. J Plant Growth Regul 26:143-159

Bell E, Creelman RA, Mullet JE (1995) A chloroplast lipoxygenase is required for wound-induced jasmonic acid accumulation in Arabidopsis. Proc Natl Acad Sci USA 92:8675-8679

Boyington JC, Gaffney BJ, Amzel LM (1997) The three-dimensional structure of soybean lipoxygenase-1: an arachidonic acid 15-lipoxygenase. Adv Exp Med Biol 400:133-138

Brash AR (1999) Lipoxygenases: occurrence, functions, catalysis, and acquisition of substrate. J Biol Chem 274:23679-23682

Caldelari D, Wang GG, Farmer EE, Dong XN (2011) Arabidopsis lox3 lox 4 double mutants are male sterile and defective in global proliferative arrest. Plant Mol Biol 75:25-33
Cao SX, Chen H, Zhang C, Tang YF, Liu JY, Qi HY (2016) Heterologous expression and biochemical characterization of two lipoxygenases in oriental melon, Cucumis melo var. makuwa Makino. PLoS ONE 11:e0153801

Chen XS, Kurre U, Jenkins NA, Copeland NG, Funk CD (1994) Cdna cloning, expression, mutagenesis of C-terminal isoleucine, genomic structure, and chromosomal localizations of murine 12-lipoxygenases. J Biol Chem 269:13979-13987

Chen GP, Hackett R, Walker D, Taylor A, Lin ZF, Grierson D (2004) Identification of a specific isoform of tomato lipoxygenase (Tomlox $C$ ) involved in the generation of fatty acid-derived flavor compounds. Plant Physiol 136:2641-2651

Chen Z, Chen X, Yan HW, Li WW, Li Y, Cai RH, Xiang Y (2015) The lipoxygenase gene family in poplar: identification, classification, and expression in response to MeJA treatment. PLoS ONE 10:e0125526

Christensen SA, Huffaker A, Kaplan F, Sims J, Ziemann S, Doehlemann G, Ji L, Schmitz RJ, Kolomiets MV, Alborn HT, Mori N, Jander G, Ni X, Sartor RC, Byers S, Abdo Z, Schmelz EA (2015) Maize death acids, 9-lipoxygenase-derived cyclopente(a)nones, display activity as cytotoxic phytoalexins and transcriptional mediators. Proc Natl Acad Sci USA 112:11407-11412

Christensen SA, Huffaker A, Hunter CT, Alborn HT, Schmelz EA (2016) A maize death acid, 10-oxo-11-phytoenoic acid, is the predominant cyclopentenone signal present during multiple stress and developmental conditions. Plant Signal Behav 11:e1120395

Coffa G, Brash AR (2004) A single active site residue directs oxygenation stereospecificity in lipoxygenases: Stereocontrol is linked to the position of oxygenation. Proc Natl Acad Sci USA 101:15579-15584

Crooks GE, Hon G, Chandonia JM, Brenner SE (2004) WebLogo: a sequence logo generator. Genome Res 14:1188-1190

Edgar RC (2004) MUSCLE: a multiple sequence alignment method with reduced time and space complexity. BMC Bioinform 5:113

Ferrie BJ, Beaudoin N, Burkhart W, Bowsher CG, Rothstein SJ (1994) The cloning of two tomato lipoxygenase genes and their differential expression during fruit ripening. Plant Physiol 106:109-118

Feussner I, Wasternack C (2002) The lipoxygenase pathway. Annu Rev Plant Biol 53:275-297

Grebner W, Stingl NE, Oenel A, Mueller MJ, Berger S (2013) Lipoxygenase6-dependent oxylipin synthesis in roots is required for abiotic and biotic stress resistance of Arabidopsis. Plant Physiol $161: 2159-2170$

Griffiths A, Barry C, Alpuche-Solis AG, Grierson D (1999) Ethylene and developmental signals regulate expression of lipoxygenase genes during tomato fruit ripening. J Exp Bot 50:793-798

Halitschke R, Baldwin IT (2003) Antisense LOX expression increases herbivore performance by decreasing defense responses and inhibiting growth-related transcriptional reorganization in Nicotiana attenuata. Plant J 36:794-807

Hall BG (2013) Building phylogenetic trees from molecular data with MEGA. Mol Biol Evol 30:1229-1235

Hickman R, Van Verk MC, Van Dijken AJH, Mendes MP, VroegopVos IA, Caarls L, Steenbergen M, Van der Nagel I, Wesselink GJ, Jironkin A, Talbot A, Rhodes J, De Vries M, Schuurink RC, Denby K, Pieterse CMJ, Wees SCM (2017) Architecture and dynamics of the jasmonic acid gene regulatory network. Plant Cell 29:2086-2105

Hornung E, Walther M, Kuhn H, Feussner I (1999) Conversion of cucumber linoleate 13-lipoxygenase to a 9-lipoxygenating species by site-directed mutagenesis. Proc Natl Acad Sci USA 96:4192-4197

Hwang IS, Hwang BK (2010) The pepper 9-lipoxygenase gene CaLOX1 functions in defense and cell death responses to microbial pathogens. Plant Physiol 152:948-967 
Kessler A (2004) Silencing the jasmonate cascade: induced plant defenses and insect populations. Science 305(2004):2042-2042

Kolomiets MV, Hannapel DJ, Chen H, Tymeson M, Gladon RJ (2001) Lipoxygenase is involved in the control of potato tuber development. Plant Cell 13:613-626

Kozlowski LP (2016) IPC—isoelectric point calculator. Biol Direct 11:55

Krieger E, Koraimann G, Vriend G (2002) Increasing the precision of comparative models with YASARA NOVA-a self-parameterizing force field. Proteins 47:393-402

Kulkarni M, Phalke S (2009) Evaluating variability of root size system and its constitutive traits in hot pepper (Capsicum annum L.) under water stress. Sci Hortic 120:159-166

Kumar S, Stecher G, Tamura K (2016) MEGA7: molecular evolutionary genetics analysis version 7.0 for bigger datasets. Mol Biol Evol 33:1870-1874

Kurunc A, Unlukara A, Cemek B (2011) Salinity and drought affect yield response of bell pepper similarly. Acta Agric Scand B 61:514-522

Leon J, Royo J, Vancanneyt G, Sanz C, Silkowski H, Griffiths G, Sanchez-Serrano JJ (2002) Lipoxygenase H1 gene silencing reveals a specific role in supplying fatty acid hydroperoxides for aliphatic aldehyde production. J Biol Chem 277:416-423

Liu SQ, Liu XH, Jiang LW (2011) Genome-wide identification, phylogeny and expression analysis of the lipoxygenase gene family in cucumber. Genet Mol Res 10:2613-2636

Livak KJ, Schmittgen TD (2001) Analysis of relative gene expression data using real-time quantitative PCR and the $2^{-\Delta \Delta C T}$ method. Methods 25:402-408

Losvik A, Beste L, Glinwood R, Ivarson E, Stephens J, Zhu LH, Jonsson L (2017) Overexpression and down-regulation of barley lipoxygenase LOX2.2 affects jasmonate-regulated genes and aphid fecundity. Int J Mol Sci 18:2765. https://doi.org/10.3390/ ijms 18122765

Mariutto M, Duby F, Adam A, Bureau C, Fauconnier ML, Ongena M, Thonart P, Dommes J (2011) The elicitation of a systemic resistance by Pseudomonas putida BTP1 in tomato involves the stimulation of two lipoxygenase isoforms. BMC Plant Biol 11:29

Melan MA, Dong XN, Endara ME, Davis KR, Ausubel FM, Peterman TK (1993) An Arabidopsis thaliana lipoxygenase gene can be induced by pathogens, abscisic-Acid, and methyl jasmonate. Plant Physiol 101:441-450

Nicholas KB, Nicholas HB, Deerfield DW (1997) GeneDoc: analysis and visualization of genetic variation. EMBNEW NEWS 4:14

Padilla MN, Hernandez ML, Sanz C, Martinez-Rivas JM (2009) Functional characterization of two 13-lipoxygenase genes from olive fruit in relation to the biosynthesis of volatile compounds of virgin olive oil. J Agric Food Chem 57:9097-9107

Padilla MN, Hernandez ML, Sanz C, Martinez-Rivas JM (2012) Molecular cloning, functional characterization and transcriptional regulation of a 9-lipoxygenase gene from olive. Phytochemistry 74:58-68

Pakdeevaraporn P, Wasee S, Taylor PWJ, Mongkolporn O (2005) Inheritance of resistance to anthracnose caused by Colletotrichum capsici in Capsicum. Plant Breeding 124:206-208

Podolyan A, White J, Jordan B, Winefield C (2010) Identification of the lipoxygenase gene family from Vitis vinifera and biochemical characterisation of two 13-lipoxygenases expressed in grape berries of Sauvignon Blanc. Funct Plant Biol 37:767-784

Porta H, Rocha-Sosa M (2002) Plant lipoxygenases. Physiological and molecular features. Plant Physiol 130:15-21

Qin C, Yu CS, Shen YO, Fang XD, Chen L, Min JM, Cheng JW, Zhao SC, Xu M, Luo Y, Yang YL, Wu ZM, Mao LK, Wu HY, Ling-Hu CY, Zhou HK, Lin HJ, Gonzalez-Morales S, Trejo-Saavedra DL, Tian H, Tang X, Zhao MJ, Huang ZY, Zhou AW, Yao XM, Cui JJ, Li WQ, Chen Z, Feng YQ, Niu YC, Bi SM, Yang XW, Li WP,
Cai HM, Luo XR, Montes-Hernandez S, Leyva-Gonzalez MA, Xiong ZQ, He XJ, Bai LJ, Tan S, Tang XQ, Liu D, Liu JW, Zhang SX, Chen MS, Zhang L, Zhang L, Zhang YC, Liao WQ, Zhang Y, Wang M, Lv XD, Wen B, Liu HJ, Luan HM, Zhang YG, Yang S, Wang XD, Xu JH, Li XQ, Li SC, Wang JY, Palloix A, Bosland PW, Li YR, Krogh A, Rivera-Bustamante RF, Herrera-Estrella L, Yin Y, Yu JP, Hu KL, Zhang ZM (2014) Whole-genome sequencing of cultivated and wild peppers provides insights into Capsicum domestication and specialization. Proc Natl Acad Sci USA 111:5135-5140

Roy A, Kucukural A, Zhang Y (2010) I-TASSER: a unified platform for automated protein structure and function prediction. Nat Protoc $5: 725-738$

Royo J, Vancanneyt G, Perez AG, Sanz C, Stormann K, Rosahl S, SanchezSerrano JJ (1996) Characterization of three potato lipoxygenases with distinct enzymatic activities and different organspecific and wound-regulated expression patterns. J Biol Chem 271:21012-21019

Sarde SJ, Bouwmeester K, Venegas-Molina J, David A, Boland W, Dicke M (2018) CaLOX2 in sweet pepper involved in jasmonatedependent induced defence against Western flower thrips. J Integr Plant Biol (In review)

Shen JY, Tieman D, Jones JB, Taylor MG, Schmelz E, Huffaker A, Bies D, Chen KS, Klee HJ (2014) A 13-lipoxygenase, TomloxC, is essential for synthesis of C5 flavour volatiles in tomato. J Exp Bot 65:419-428

Shibata D, Axelrod B (1995) Plant lipoxygenases. J Lipid Mediat Cell 12:213-228

Shin JH, Van K, Kim DH, Do Kim K, Jang YE, Choi BS, Kim MY, Lee SH (2008) The lipoxygenase gene family: a genomic fossil of shared polyploidy between Glycine max and Medicago truncatula. BMC Plant Biol 8:133

Shipp JL, Hao X, Papadopoulos AP, Binns MR (1998) Impact of western flower thrips (Thysanoptera: Thripidae) on growth, photosynthesis and productivity of greenhouse sweet pepper. Sci Hortic 72:87-102

Sloane DL, Leung R, Craik CS, Sigal E (1991) A primary determinant for lipoxygenase positional specificity. Nature 354:149-152

Steczko J, Donoho GP, Clemens JC, Dixon JE, Axelrod B (1992) Conserved histidine-residues in soybean lipoxygenase-functional consequences of their replacement. Biochemistry 31:4053-4057

Steenbergen M, Abd-el-Haliem A, Bleeker P, Dicke M, Escobar-Bravo R, Cheng G, Haring MA, Kant MR, Kappers I, Klinkhamer PGL, Leiss KA, Legarrea S, Macel M, Mouden S, Pieterse CMJ, Sarde SJ, Schuurink RC, De Vos M, Van Wees SCM, Broekgaarden C (2018) Thrips advisor: exploiting thrips-induced defences to combat pests on crops. J Exp Bot 69:1837-1848

ul Hassan MN, Zainal Z, Ismail I (2015) Green leaf volatiles: biosynthesis, biological functions and their applications in biotechnology. Plant Biotechnol J 13:727-739

Umate P (2011) Genome-wide analysis of lipoxygenase gene family in Arabidopsis and rice. Plant Signal Behav 6:335-338

Vandesompele J, De Preter K, Pattyn F, Poppe B, Van Roy N, De Paepe A, Speleman F (2002) Accurate normalization of real-time quantitative RT-PCR data by geometric averaging of multiple internal control genes. Genome Biol 3:RESEARCH0034

VanDoorn A, Kallenbach M, Borquez AA, Baldwin IT, Bonaventure G (2010) Rapid modification of the insect elicitor N-linolenoylglutamate via a lipoxygenase-mediated mechanism on Nicotiana attenuata leaves. BMC Plant Biol 10:164

Vellosillo T, Martinez M, Lopez MA, Vicente J, Cascon T, Dolan L, Hamberg M, Castresana C (2007) Oxylipins produced by the 9-lipoxygenase pathway in Arabidopsis regulate lateral root development and defense responses through a specific signaling cascade. Plant Cell 19:831-846 
Vogt J, Schiller D, Ulrich D, Schwab W, Dunemann F (2013) Identification of lipoxygenase (LOX) genes putatively involved in fruit flavour formation in apple (Malus $x$ domestica). Tree Genet Genomes 9:1493-1511

Yan LH, Zhai QZ, Wei JN, Li SY, Wang B, Huang TT, Du MM, Sun JQ, Kang L, Li CB, Li CY (2013) Role of tomato lipoxygenase D in wound-induced jasmonate biosynthesis and plant immunity to insect herbivores. PLoS Genet 9:e1003964

Yates A, Akanni W, Amode MR, Barrell D, Billis K, Carvalho-Silva D, Cummins C, Clapham P, Fitzgerald S, Gil L, Giron CG, Gordon L, Hourlier T, Hunt SE, Janacek SH, Johnson N, Juettemann T, Keenan S, Lavidas I, Martin FJ, Maurel T, McLaren W, Murphy DN, Nag R, Nuhn M, Parker A, Patricio M, Pignatelli M, Rahtz M, Riat HS, Sheppard D, Taylor K, Thormann A, Vullo A,
Wilder SP, Zadissa A, Birney E, Harrow J, Muffato M, Perry E, Ruffier M, Spudich G, Trevanion SJ, Cunningham F, Aken BL, Zerbino DR, Flicek P (2016) Ensembl 2016. Nucleic Acids Res 44:D710-D716

Zhang B, Chen KS, Bowen J, Allan A, Espley R, Karunairetnam S, Ferguson I (2006) Differential expression within the LOX gene family in ripening kiwifruit. J Exp Bot 57:3825-3836

Zhang B, Yin XR, Li X, Yang SL, Ferguson IB, Chen KS (2009) Lipoxygenase gene expression in ripening kiwifruit in relation to ethylene and aroma production. J Agric Food Chem 57:2875-2881

Zhang C, Jin YZ, Liu JY, Tang YF, Cao SX, Qi HY (2014) The phylogeny and expression profiles of the lipoxygenase $(L O X)$ family genes in the melon (Cucumis melo L.) genome. Sci Hortic 170:94-102 